\title{
Is impaired joint attention present in non- clinical individuals with high autistic traits?
}

\author{
Shuo Zhao ${ }^{1,2,3^{*}}$, Shota Uono ${ }^{4}$, Sayaka Yoshimura ${ }^{4}$ and Motomi Toichi ${ }^{1,2}$
}

\begin{abstract}
Background: Joint attention skills are impaired in individuals with autism spectrum disorder (ASD). Recently, varying degrees of autistic social attention deficit have been detected in the general population. We investigated gaze-triggered attention in individuals with high and low levels of autistic traits under visual-auditory cross-modal conditions, which are more sensitive to social attention deficits than unimodal paradigms.

Methods: Sixty-six typically developing adults were divided into low- and high-autistic-trait groups according to scores on the autism-spectrum quotient (AQ) questionnaire. We examined gaze-triggered attention under visual-auditory cross-modal conditions. Two sounds (a social voice and a non-social tone) were manipulated as targets to infer the relationship between the cue and the target. Two types of stimulus onset asynchrony (SOA) conditions (a shorter 200-ms SOA and a longer 800-ms SOA) were used to directly test the effect of gaze cues on the detection of a sound target across different temporal intervals.
\end{abstract}

Results: Individuals with high autistic traits (high-AQ group) did not differ from those with low autistic traits (low-AQ group) with respect to gaze-triggered attention when voices or tones were used as targets under the shorter SOA condition. In contrast, under the longer SOA condition, gaze-triggered attention was not observed in response to tonal targets among individuals in the high-AQ group, whereas it was observed among individuals in the low-AQ group. The results demonstrated that cross-modal gaze-triggered attention is short-lived in individuals with high autistic traits.

Conclusions: This finding provides insight into the cross-modal joint attention function among individuals along the autism spectrum from low autistic traits to ASD and may further our understanding of social behaviours among individuals at different places along the autistic trait continuum.

Keywords: Autism-spectrum quotient (AQ), Joint attention, Low autistic traits, High autistic traits, Cross-modal

\section{Background}

One of the key core features of social interactions involves detecting other people's desires, intentions, and mental states, which helps us to understand their behaviour and the reasons for their actions. The ability to coordinate attention to focus on the same location or event as another person, the phenomenon known as joint attention [1], has been thought to be a precursor of the development of the ability to attribute mental states

\footnotetext{
*Correspondence: zhaoshuo09@gmail.com

${ }^{1}$ Faculty of Human Health Science, Graduate School of Medicine, Kyoto University, 53 Shogoin Kawahara-cho, Sakyo-ku, Kyoto 606-8507, Japan ${ }^{2}$ International Research Fellow of the Japan Society for the Promotion of Science, Tokyo, Japan

Full list of author information is available at the end of the article
}

(i.e. theory of mind). This phenomenon can be achieved when individual $\mathrm{B}$ perceives individual A's direction of attention. Individual B then orients his/her attention to the same object or event. Individuals $\mathrm{A}$ and $\mathrm{B}$ are now attending to the same object, based on individual B using the attention cues of individual A [2]. In the context of joint attention, developing children typically use the eye gaze of others to make attributions about other people's cognitive states, such as their intentions, and to speculate about what they want to do $[1,3]$.

During the past two decades, cognitive psychologists have focused on the social attentional mechanisms of joint attention (e.g. [4-7]). Researchers have commonly investigated gaze-triggered attention using a modification of the 
Posner cueing paradigm [8]. For instance, in one of the pioneering studies [9], subjects observed an unpredictable, directed eye gaze towards the right or left as a cue, and a letter target was subsequently presented either in the gazed-at or in the opposite direction. Subjects were asked to respond as quickly and as accurately as possible, and their reaction times (RTs) were measured. The RTs for detecting the letter target were faster when it appeared in the same direction as the cues than when it appeared in the opposite direction. This finding indicated that gaze direction reflexively triggers the observer's attentional orientation. This attentional effect was also found under visual-auditory cross-modal conditions [10] (i.e. using eye gaze as the cue and a tone as the target).

Recently, individual differences in social attention have been identified, particularly in those with autism spectrum disorder (ASD) [11-13]. A lack of joint attention in individuals with ASD has been well documented in the clinical literature [14], and it has attracted attention as an early marker of ASD (e.g. [15-19]; see review in [20]). Furthermore, in our previous study [13], we found that gaze-triggered attention was also impaired under visualauditory cross-modal conditions in adults with ASD. Additionally, several research studies have suggested that autistic social attention deficiencies may also be found, to different degrees, throughout the general population [21, 22]. It has been suggested that no clear boundary separates normal from psychopathological and that ASD is merely at one extreme end of a continuum [23, 24]. It is, therefore, important to evaluate the distribution of gazetriggered attention in typical individuals, which might reflect developmental variety in social cognition.

Previous studies [25-27] have demonstrated that autistic traits affect gaze-triggered attention. These studies have generally administered the autism-spectrum quotient (AQ) questionnaire to measure autistic traits in the population at large [23]. For instance, Bayliss and colleagues reported a negative correlation between AQ scores and the magnitude of the gaze-cueing effect [25] and revealed that the effect of target context (i.e. scrambled vs. normal face) on gaze-triggered attention differed as a function of an individual's placement on the autistic spectrum. In particular, a greater gaze-triggered attention effect was observed for a scrambled face context compared with a normal face context among individuals with a high level of autistic traits, suggesting that this bias was related to the level of attention to detail [26]. Moreover, Hudson et al. [27] found a smaller cueing effect in response to gaze in antisocial individuals than in prosocial individuals within a low-AQ group; the effect was equal in both cue within the high-AQ group. All these studies used visual cues and visual targets under a unimodal condition. However, real life includes various environmental stimuli, including sounds. We need to constantly coordinate our attention with that of others by using cues and targets that belong to different modalities; therefore, it is also necessary to investigate the underlying mechanisms of how we modulate the effects of gaze-triggered attention under cross-modal conditions and to examine how these vary with autism traits and relate to a diagnosis of autism.

We attempted to extend the previous research in the following respects. First, the experimental paradigms used in previous studies [25-27] have not been applied to gaze-triggered attention in ASD individuals, resulting in a lack of evidence of the overall variation in gazetriggered attention associated with autistic traits, including ASD. Second, most of the previous studies found intact gaze-triggered attention when visual cues and targets were used under unimodal conditions ([28-30]; for a review, see [31]). In contrast, our previous study [13] clearly showed that gaze-triggered attention was impaired in individuals with ASD under cross-modal conditions. Gaze-triggered attention was impaired in individuals with ASD when the cue-target relationship was weak (i.e. a social gaze cue and a non-social tone target), whereas it was unimpaired when there was a strong cue-target relationship (i.e. a social gaze cue and a social voice target). Hence, to bridge the gap between our understanding of the gaze-triggered attention of typically developing individuals and that of the gaze-triggered attention of individuals with a diagnosis of ASD, this paper focuses on the gaze-triggered attention of individuals with autistic traits in response to a visual cue and an auditory target (i.e. cross-modal conditions).

In this study, we first examined gaze-triggered attention under visual-auditory cross-modal conditions. Two sounds (a social voice and a non-social tone) were manipulated as targets to infer the relationship between the cue and target. The previous study [13] indicated that the effect of gaze cues on individuals with ASD could be mediated by different stimulus onset asynchronies (SOAs) between the auditory target and the gaze cue (i.e. gaze-triggered attention was observed when the target was a voice at a shorter, 200-ms, SOA, whereas the effect was not observed at a longer, 800-ms, SOA). Thus, to test the effect of gaze cues on the detection of a sound target, we used two types of SOA. Participants were asked to identify the direction of an auditory target as accurately and rapidly as possible following a gaze cue. All participants were divided into low- or highautistic-trait groups according to AQ score. Based on the previous study [13], we hypothesised that individuals with high autistic traits would be impaired with respect to gaze-triggered attention when the cue-target relationship was weak (i.e. a social gaze cue and a non-social tone target). In contrast, we predicted that the gazetriggered attention of individuals with low autistic traits 
would be intact even when the cue-target relationship was weak. Taken together, the aims of the study were as follows: (1) to investigate whether visual-auditory crossmodal gaze-triggered attention is impaired in individuals with high autistic traits and (2) to examine whether the visual-auditory cross-modal gaze-triggered attention of these individuals is impaired when the cue-target relationship is weak (i.e. a social gaze cue and a nonsocial tone target).

\section{Methods}

\section{Ethics statement}

The experimental procedures were approved by the local ethics committee of the Graduate School and Faculty of Medicine at Kyoto University. There were no foreseeable risks to the participants, and no personally identifying information was collected. Participants provided background information and gave written informed consent. The procedures complied with the ethical standard of the 1964 Declaration of Helsinki regarding the treatment of human participants in research.

\section{Participants}

Sixty-six naïve participants (mean age $=20.67 \pm 0.966$ standard deviation $(S D)$ years; 41 women, 25 men) recruited from Kyoto University participated in the experiment for payment. All participants were right-handed, as assessed by the Edinburgh Handedness Inventory [32], and had normal or corrected-to-normal visual and auditory acuity.

\section{Apparatus}

Stimulus presentation and data acquisition were controlled using Presentation (NeuroBehavioral Systems) on a Windows computer. Stimuli were presented on a 19-in. monitor (Dell; screen resolution $1024 \times 768$ pixels; refresh rate $60 \mathrm{~Hz}$ ). The distance between the monitor and the participants was fixed at approximately $57 \mathrm{~cm}$ using a headrest. All auditory stimuli were presented through headphones.

\section{Stimuli}

Consistent with the previous study, the same neutral face of a female model (MO) was selected from Ekman and Friesen [33]. The gaze direction was then manipulated. The irises and pupils of the eyes were cut from the original photographs and pasted to fit over the right or left side of the eyes using Photoshop 5.0 (Adobe). We cropped the photographs in an ellipse $8.3^{\circ}$ wide and $12.1^{\circ}$ high to exclude hair and background.

We used two types of auditory stimuli as targets. One was sampled from a native Japanese woman: an /i/ voice sound (F0 frequency of $300 \mathrm{~Hz}, 80 \mathrm{~dB}$ sound pressure level (SPL)), which is similar to the /iy/ sound in English.
The other was a pure tone of a voice with a frequency similar to F0 (300 Hz, $80 \mathrm{~dB} \mathrm{SPL})$, which was produced using Audacity V1.3.13 software (AudacityStore.com). Both auditory targets had a duration of $150 \mathrm{~ms}$.

\section{Design}

The experiment was constructed as a four-factorial, mixed randomised-repeated design, with auditory (voice sound or tone sound), validity (valid, invalid), and SOA (200, $800 \mathrm{~ms}$ ) as repeated factors and group (low- or highAQ group) as the randomised factor.

\section{Measures}

We used a Japanese version of the AQ [34], which is a 50 -item self-rated scale designed to measure each of five domains of interest: social skill, communication, imagination, attention to detail, and attention-switching. Ten statements were used to measure each of the assessed traits, and participants were asked whether they 'strongly agree', 'slightly agree', 'slightly disagree', or 'strongly disagree' with each statement. Participants with a high score in the AQ possessed a greater number of autistic traits. The AQ score has been shown to have good test-retest reliability, good internal consistency, and acceptably high sensitivity and specificity [23]. In this sample, the mean AQ score was $19.44 \pm 7.06 S D$ of 50 . Based on previous research [27], participants scoring lower than the median AQ score of 18.5 were assigned to the lowAQ group $(M=13.64, S D=3.92$, range $=5-18, N=33)$, whereas those scoring higher than the median AQ score of 18.5 were assigned to the high-AQ group $(M=25.24$, $S D=4.05$, range $=19-35, N=33$ ). Kurita et al. [34] reported a cut-off of 32 for screening adults with highfunctioning pervasive developmental disorders (PDD) using a Japanese version of the AQ (AQ-J). In this study, we considered individuals in the high-AQ group to have milder autistic traits than those diagnosed with ASD. The gender ratio in the two groups did not significantly differ (12 men and 21 women in the low-AQ group, 13 men and 20 women in the high-AQ group, Fisher's exact test, $p>0.1$.

\section{Procedure}

We used the same experimental paradigm as in our previous study [13] (see Fig. 1). For each trial, a fixation cross point was first presented in the centre of the screen for $600 \mathrm{~ms}$. A neutral face with a straight gaze was then presented at this location as a background. After $500 \mathrm{~ms}$, a neutral facial cue with the eye gaze directed right or left was presented in the centre of the screen. The SOA between the auditory target and gaze cue was 200 or $800 \mathrm{~ms}$. The SOA condition was determined by randomising each auditory target condition to exclude an effect specific to a sequence of 


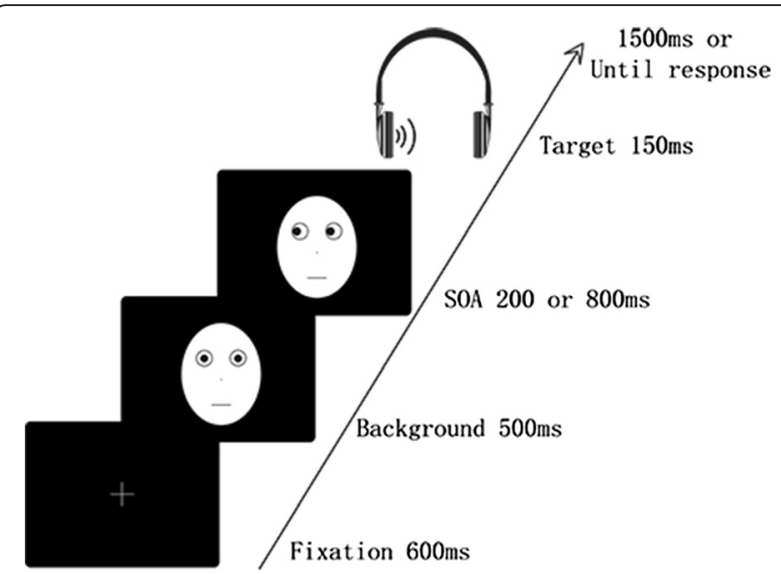

Fig. 1 Illustrations of stimulus presentations. Actual stimuli were photographs of faces (see Figure 1 in [33])

SOA conditions. Subsequently, an auditory stimulus target (voice sound or tone sound) was presented in the left or right ear through headphones for $150 \mathrm{~ms}$. Participants were asked to communicate as quickly and exactly as possible whether the target was presented to the left or to the right ear by pressing the corresponding key on the switch key using the index or middle finger of their dominant hand, respectively. Response time (RT) was measured in each trial. The gaze cue remained visible until the response or until 1,500 ms had elapsed. The targets appeared randomly on the same or opposite side as the gaze direction when the eyes looked left or right. If participants could not respond in a trial, the data were excluded as incorrect. The target appeared in the cued location in $50 \%$ of the trials. Participants were told that the cues did not predict the target location and were instructed to fix on the centre of the screen in each trial.

The experiment consisted of eight blocks of 52 trials, including 32 catch trials in which the target did not appear. Forty-eight trials were performed under each condition. Each condition was presented in a pseudorandom order. Participants were allowed to rest between blocks. Fifty-two practice trials preceded the experimental trials. At the end of the experiment, all participants completed the AQ questionnaire.

\section{Analysis}

The data were analysed using IBM's SPSS Statistics software. Incorrect responses and responses of $<150 \mathrm{~ms}$ or $>1000 \mathrm{~ms}(0.24 \%$ of the trials) were excluded from the RT analysis. The mean RT under each condition was calculated for each participant. First, trials with RTs faster than $150 \mathrm{~ms}$ or slower than $1000 \mathrm{~ms}$ (low-AQ group: $0.17 \%$ of trials, high-AQ group: $0.32 \%$ of trials) and those with incorrect responses (low-AQ group:
$1.77 \%$ of trials, high-AQ group: $1.96 \%$ of trials) were excluded from the RT analysis.

Then, the mean RT of all participants was $\log _{10^{-}}$ transformed and submitted to a four-way repeatedmeasures analysis of variance (ANOVA) with validity (valid, invalid), auditory target (voice, tone), and SOA $(200,800 \mathrm{~ms})$ as within-participant factors, and group (low $A Q$, high $A Q$ ) as the between-participant factor. Data from significant interactions were analysed separately, using three-way ANOVAs for 200 and $800 \mathrm{~ms}$ SOA. To examine whether two three-way ANOVAs were significant for the interaction, if present, follow-up simple effect analyses were conducted. Furthermore, a preliminary analysis of covariance (ANCOVA), using participants' gender as a covariate, revealed that gender did not affect the group $x$ validity $\times$ target $\times$ SOA interaction.

\section{Results}

\section{Cueing effects on accuracy}

There was no significant difference in the error rates of the low- and high-AQ groups $(F(1,64)=0.14, p=$ $0.71, \eta \mathrm{p} 2=0.002)$. We found a significant main effect of validity $(F(1,64)=30.19, p<0.001, \eta \mathrm{p} 2=0.32)$, with fewer incorrect responses under the valid compared with the invalid condition $(1.3$ vs. $2.5 \%)$ and of SOA $(F(1,64)=33.32, p<0.001, \eta \mathrm{p} 2=0.34)$ and with more incorrect responses under the shorter compared with the longer SOA condition (2.4 vs. $1.4 \%)$; we also found a significant interaction of validity $\times$ SOA $(F(1,64)=11.34, p=0.001, \eta \mathrm{p} 2=0.015)$, indicating fewer incorrect responses in valid compared with invalid ones under the shorter SOA (1.3 vs. $3.4 \%$ ) but not under the longer SOA (1.2 vs. $1.6 \%$ ) condition.

\section{Cueing effects on reaction times}

We first investigated whether the data set was wellmodelled by a normal distribution in all of the conditions. However, when using tone and validity at the $800 \mathrm{~ms}$ SOA condition, the data were not normally distributed (Shapiro-Wilk: $p=0.04$ ). Therefore, the mean RT data were logarithmically transformed and analysed using a four-way ANOVA. We conducted a two-group (low-, high-AQ group) $\times$ two-target (voice, tone) $\times$ twovalidity (valid, invalid) $\times$ two-SOA $(200,800 \mathrm{~ms})$ analysis. The ANOVA revealed a significant main effect of validity $(F(1,64)=51.65, p<0.001, \eta p 2=0.45)$, with faster responses under the valid than the invalid condition. There was no significant main effect of SOA $(F(1,64)=$ $1.02, p=0.32, \eta \mathrm{p} 2=0.016)$, target $(F(1,64)=3.74, p=$ $0.06, \eta \mathrm{p} 2=0.06)$, or group $(F(1,64)=0.30, p=0.58$, $\eta \mathrm{p} 2=0.005)$, suggesting that the perceptual salience of the two types of stimuli, and the perceptual response in each group, did not differ. Significant interactions of validity $\times \operatorname{SOA}(F(1,64)=97.34, p<0.001, \eta \mathrm{p} 2=0.60)$, 
target $\times$ validity $\times$ SOA $(F(1,64)=7.76, p=0.007, \eta \mathrm{p} 2=$ $0.108)$, and group $\times$ target $\times$ validity $\times \operatorname{SOA}(F(1,64)=$ $4.35, p=0.04, \eta \mathrm{p} 2=0.06)$ were also found.

No difference of gaze-triggered attention under the short (200 ms) SOA condition between the low- and high-AQ groups Because we found significant interactions among the four factors, the data were analysed separately for the 200- and 800-ms SOA conditions. A three-way ANOVA of the RT (logarithmically transformed) data under the 200-ms SOA condition (Fig. 2a, Table 1a) revealed a significant main effect of validity $(F$ $(1,64)=100.85, p<0.001)$, with faster responses under valid than invalid conditions, and a marginally significant effect of target $(F(1,64)=8.18, p=0.06)$, with faster responses under the voice than the tone condition; however, there was no significant effect involving group ( $F$ (1, $64)=0.42, p=0.52)$. Interestingly, there was a significant interaction of target $\times$ validity $(\mathrm{F}(1,64)=5.40, p=0.02)$. A post hoc test revealed a significant validity effect (both of $p<0.001)$ using voice and tone as targets, indicating that gaze-triggered attention was elicited using voice and tone as targets in both the low- and high-AQ groups. Furthermore, a significant difference was found with the valid condition between voice and tone as targets across groups $(p=0.01)$, suggesting that gaze-triggered attention was elicited more rapidly when using voice rather than tone targets. These results reveal that gaze-triggered attention to a voice target was enhanced in both the low- and highAQ groups under the shorter SOA condition.

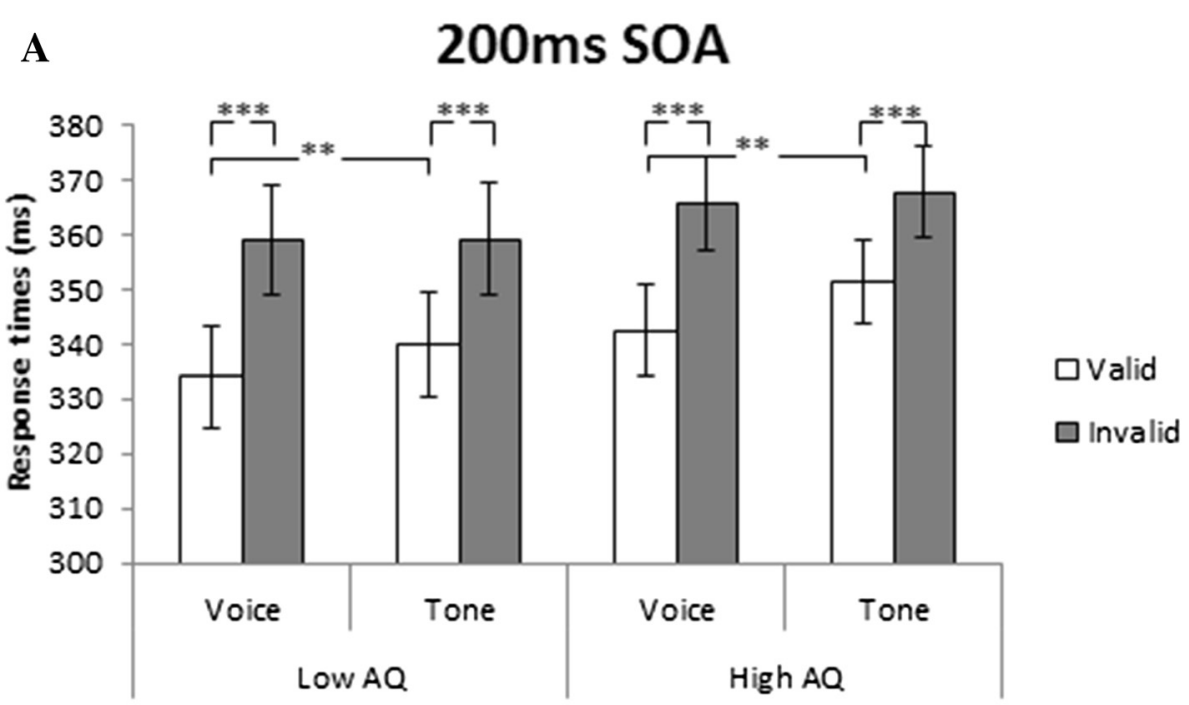

B $\quad 800 m s$ SOA

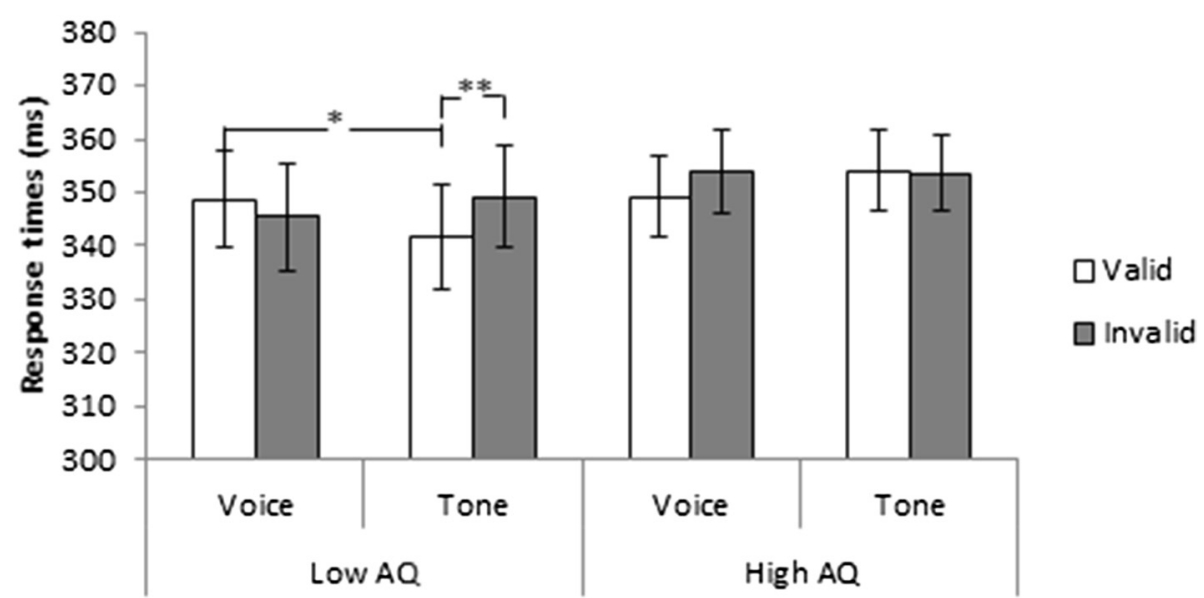

Fig. 2 Effect of gaze direction on response times (RTs) under each condition with $200 \mathrm{~ms}$ (a) and $800 \mathrm{~ms}$ (b) SOA. Error bars represent standard errors of the mean (SEMs). ${ }^{* *} p<0.001,{ }^{* *} p<0.01,{ }^{*} p<0.05$ 
Table 1 Mean low- and high-AQ group response times (ms) to auditory targets according to validity and stimulus onset asynchrony

\begin{tabular}{|c|c|c|c|c|}
\hline \multirow[t]{2}{*}{ Auditory target } & \multicolumn{2}{|l|}{ Voice } & \multicolumn{2}{|l|}{ Tone } \\
\hline & Valid & Invalid & Valid & Invalid \\
\hline \multicolumn{5}{|l|}{$\begin{array}{l}\text { Stimulus onset } \\
\text { asynchrony }\end{array}$} \\
\hline \multicolumn{5}{|l|}{ (a) $200 \mathrm{~ms}$ (SEM) } \\
\hline Low-AQ group & 333.9 (8.3) & $358.8(8.4)$ & $339.6(7.6)$ & $359.1(8.4)$ \\
\hline High-AQ group & $342.4(9.3)$ & 365.5 (9.9) & $351.3(9.5)$ & $367.6(10.1)$ \\
\hline \multicolumn{5}{|l|}{ (b) $800 \mathrm{~ms}$ (SEM) } \\
\hline Low-AQ group & $348.6(7.6)$ & $345.4(7.8)$ & 341.7 (7.6) & $349.2(7.1)$ \\
\hline High-AQ group & $349.2(9.1)$ & $354.0(9.9)$ & $354.0(9.7)$ & $353.6(9.6)$ \\
\hline
\end{tabular}

Impaired gaze-triggered attention under the long (800 ms) SOA condition in the high-AQ group In terms of the 800-ms SOA condition (Fig. 2b, Table 1b), a three-way ANOVA of the RT (logarithmically transformed) data revealed no significant main effect with respect to target $(F(1,64)=0.05)$, validity $(F(1,64)=1.77)$, or group $(F(1,64)=0.18)(p>0.1$ for all). More importantly, there was a significant interaction of target $\times$ validity $\times$ group $(F(1,64)=7.07, p=0.01)$. A post hoc test revealed a significant validity effect using tone as the target in participants with low-AQ scores $(p=0.04)$, indicating that gaze-triggered attention was elicited when using tones as targets. Furthermore, a significant difference was found between voice and tone targets under the valid condition in the low-AQ group $(p=0.03)$, suggesting that inhibition of return (IOR) was faster for voice than tone targets in participants with low-AQ scores. In contrast, no significant difference was found for validity or targets in participants with high-AQ scores (all $p>0.1$ ). These results showed that gaze-triggered attention was observed under the longer SOA condition in participants with low-AQ scores, but not in participants with high-AQ scores.

\section{Discussion}

This study manipulated gaze-triggered attention using sound targets (i.e. voice and tone) and SOA (200 and $800 \mathrm{~ms}$ ) under visual-auditory cross-modal conditions to examine whether this capacity is related to the extent of autistic-like traits, measured with the AQ. The gazetriggered attention of individuals with high levels of autistic traits (the high-AQ group) did not differ from that of individuals with low levels of autistic traits when voice or tone was used as a target under the shorter SOA condition. In particular, voice but not tone facilitated gaze-triggered attention in both low- and high-AQ groups. In contrast, the low-AQ but not the high-AQ group exhibited significant gaze-triggered attention to a tone target under the longer SOA condition. This result suggests that the gaze-triggered attention of individuals with high autistic traits was short-lived in response to a sound stimulus.

\section{Similar gaze-triggered attention between individuals with low and high autistic traits at the shorter SOA}

Previous findings [13] were replicated in this study, as we observed that a shorter SOA facilitated gaze-triggered attention more rapidly to voice than tone targets in individuals both low and high in autistic traits. Previous studies of normal individuals [35-40] demonstrated that gaze-triggered attention is facilitated when the cue and target are congruent (e.g. a happy face as a cue and a pleasant infant as the target). Consistent with these previous studies, a higher level of gaze-triggered attention was found in both groups under the shorter SOA condition when the cue-target relationship was congruent (i.e. a social cue and a social target) than when it was incongruent (i.e. a social cue and a non-social target). Although our previous study of individuals with ASD [13] also demonstrated the same pattern of gaze-triggered attention under the shorter SOA condition, it did not reveal a significant gaze-cueing effect when there was a weak cuetarget relationship (i.e. a social cue and a non-social target). Based on these findings, the results suggest that gaze-triggered attention is modulated by the cue-target relationship in individuals across the autism spectrum and that it is intact in normal participants, even when the cue-target relationship is weak.

Impairment in gaze-triggered attention in individuals with high autistic traits at the longer SOA

Under the longer SOA condition, individuals with low autistic traits showed a significant gaze-cueing effect in response to a tone but not to a voice target. Previous studies $[13,41]$ have suggested that IOR was modulated by the cue-target relationship in typical individuals. These studies found that an IOR effect occurred earlier during the time course of SOA when the contextual cue-target relationship was strong (i.e. a social cue and a social target) than when it was weak (i.e. a social cue and a non-social target). Consistent with these findings $[13,41]$, the present study observed a faster IOR of gaze-triggered attention in individuals with low autistic traits when the cue-target relationship was strong (i.e. congruence between the social gaze cue and the social voice target) than when the cue-target relationship was weak (i.e. incongruence between the social gaze cue and the non-social tone target) under the longer SOA conditions. In contrast, in individuals with high autistic traits, gaze-triggered attention was not observed in response to voice and tone targets at the longer SOA. These results suggest that individuals with high levels of autistic traits do not demonstrate contextual modulation of the IOR 
effect and that the cueing effect for both kinds of targets is diminished at the intermediate SOA. Moreover, a previous study [42] showed that the IOR effect occurred earlier in individuals with ASD than in normal individuals when using non-predictive target-peripheral cues during the time course of SOA; the IOR effect was present at the shorter SOA (300 ms) in individuals with ASD and at the longer SOA (500-700 ms) in normal individuals. Based on this study, the impairment in gazetriggered attention to tone targets at the longer SOA might also be caused by the early-onset appearance of IOR in individuals with high autistic traits.

Zhao et al. [13] proposed that gaze-triggered attention is likely to be impaired in individuals with ASD when the contextual relationship between a cue and a target is weak (i.e. a gaze cue and a tone target); gaze-triggered attention was impaired for tone targets during shorter and longer (200 and $800 \mathrm{~ms}$ ) SOAs. Whereas participants with low autistic traits showed significant gaze-triggered attention to tone targets across the time course, participants with high autistic traits, who did not meet criteria for ASD, showed impaired gaze-triggered attention to tone targets at only the longer SOA. In addition to increasing with the degree of severity of autistic traits, gaze-triggered attention was also impaired by the strength of the contextual relationship between cue and target. Given that an identical paradigm (with fewer trials) was also implemented in our previous study [13], a quantified variability of the gaze-cueing effect (i.e. the difference in score between the valid and invalid conditions) to tone targets was observed along the autistic spectrum; scores for the group with high autistic traits $(7.9 \mathrm{~ms} \pm 2.9 \mathrm{SD})$ were intermediary to the scores for the low autistic traits $(13.5 \mathrm{~ms} \pm 2.9 \mathrm{SD})$ and ASD $(1.3 \mathrm{~ms} \pm 3.9 \mathrm{SD})$ groups. Thus, we suggest that the degree of impairment in gazetriggered attention varies with autistic traits. These findings might contribute to understanding the continuum that includes individuals with low and high autistic traits and diagnoses of ASD in terms of cross-modal joint attention and might improve our understanding of social behaviours among individuals along the autistic spectrum.

\section{Implications of the development of social cognition}

The pattern of gaze-triggered attention shown by participants with low and high levels of autistic traits differed. This finding might reflect developmental variety in social cognition within the general population. If differences regarding the processing of social information emerged in infancy, they may continue to affect cognitive abilities into adulthood. Importantly, the attention with gaze directional cues to a specific target shown by individuals with high autistic traits did not endure. Mundy and colleagues [43-45] proposed that joint attention enhances the processing of information, including others' internal states and attended objects, and that individuals with autism do not receive the benefits of enhanced information processing through joint attention with others. Consistent with the prediction of this model, previous studies have found that typically developing individuals show a greater resistance to IOR that emerges in social (i.e. $800 \mathrm{~ms}$ ) than in non-social directional cueing (i.e. $300 \mathrm{~ms}$ ) [46, 47]; this suggests that information processing of an attended object is more effective with social directional cues than with non-social directional cues. However, the current study speculated on an early-onset of IOR to social cues, even in typically developing individuals with high autistic traits. Another study found that, under a joint attention context, gaze allocation to another's eye gaze was higher for directed targets than non-directed targets in individuals with low levels of autistic traits; no differences were observed in individuals with high levels of autistic traits [48]. Based on the evidence, information processing of attended objects under the context of joint attention may be less enhanced in typically developing individuals with high autistic traits than in those with low autistic traits. Furthermore, given that joint attention is a precursor to theory of mind [49], impaired gaze-triggered attention might impede and differentially affect the development of the ability to understand others' mental states (beliefs, desires, intentions, imagination, emotion, etc.) of individuals with high autistic traits. Hence, the findings of the current study may improve our understanding of the social behaviours of people who have high autistic traits without a diagnosis of ASD.

\section{Limitations in the current study}

First, given that the $\mathrm{AQ}$ is a continuous measure, it is a very interesting question whether there is a correlation between AQ score and gaze-cueing effect. However, we did not find a significant correlation with AQ scores in the magnitude of gaze-cueing effect (all $p>0.1$ ). One possible explanation is that in individuals with intermediate autistic traits, gaze-cueing effect may be modulated less by autistic traits and more by general individual differences in, for example, IQ or motor performance. These data were not collected in our study, which presents a limitation of the findings. Second, the stimulus presentation consisted of a passive viewing paradigm. However, in real life, social interactions are typically characterised by reciprocity and interdependence of behaviours rather than simple passive stimulus-response patterns. Recent developments in social cognition research have moved towards using socially responsive agents [50-53] to investigate social attention under an ecologically valid context. Future research may benefit from investigations of the initiation and response to gaze-triggered attention in individuals 
along the autistic trait spectrum using interactiveresponse patterns such as gaze-contingent stimuli.

\section{Conclusions}

Our results demonstrated that gaze-triggered attention was impaired in response to tone targets at the longer SOA in individuals with high autistic traits, whereas their gaze-triggered attention to voice and tone targets was intact at the shorter SOA, indicating that gaze-triggered attention was short-lived and reduced in individuals with high autistic traits. More generally, our findings highlight the importance of the overall variation in gaze-triggered attention among those with autistic traits, including individuals with ASD, and underscores the fact that the degree of impairment in gaze-triggered attention varies as a function of autistic traits. Thus, this research may offer a new perspective on the social cognition of individuals along the autistic spectrum and help future researchers carefully consider their recruitment strategy, particularly when investigating certain gaze-triggered attention tasks.

\section{Abbreviations}

AQ: autism-spectrum quotient; ASD: autism spectrum disorder.

\section{Competing interests}

The authors declare that they have no competing interests.

\section{Authors' contributions}

SZ collected and analysed the data and prepared the figures. SZ, SU, SY, and MT designed the experiment and wrote the manuscript. All authors read and approved the manuscript.

\section{Acknowledgements}

We would like to acknowledge and thank the subjects involved in the study. This study was supported by a Grant-in-Aid for Overseas JSPS Fellows (15F15307), the Organization for Promoting Developmental Disorder Research.

\section{Author details}

${ }^{1}$ Faculty of Human Health Science, Graduate School of Medicine, Kyoto University, 53 Shogoin Kawahara-cho, Sakyo-ku, Kyoto 606-8507, Japan. ${ }^{2}$ International Research Fellow of the Japan Society for the Promotion of Science, Tokyo, Japan. ${ }^{3}$ Organization for Promoting Developmental Disorder Research, Kyoto, Japan. ${ }^{4}$ Department of Neurodevelopmental Psychiatry, Habilitation and Rehabilitation, Graduate School of Medicine, Kyoto University, Kyoto, Japan.

Received: 29 July 2015 Accepted: 4 December 2015

Published online: 22 December 2015

\section{References}

1. Baron-Cohen S, Campbell R, Karmiloff-Smith A, Grant J, Walker J. Are children with autism blind to the mentalistic significance of the eyes? $\mathrm{Br} J$ Dev Psychol. 1995;13:379-98.

2. Emery NJ. The eyes have it: the neurothology, function and evolution of social gaze. Neurosci Biohehav Rev. 2000;24:581-604.

3. Freire A, Eskritt M, Lee K. Are eyes windows to a deceiver's soul? Children's use of another's eye gaze cues in a deceptive situation. Dev Psychol. 2004;40:1093-104.

4. Baron-Cohen S. The eye direction detector (EDD) and the shared attention mechanism (SAM): two cases for evolutionary psychology. In: Moore C, Dunhan PJ, editors. Joint attention: its origins and role in development. Hillsdale: Erlbaum; 1995. p. 41-59.

5. Farroni T, Johnson MH, Csibra G. Mechanisms of eye gaze perception during infancy. Cogn Neurosci. 2004;16:1320-6.
6. Frischen A, Bayliss AP, Tipper SP. Gaze cueing of attention: visual attention, social cognition, and individual differences. Psychol Bull. 2007;133:694-724.

7. Mundy P, Newell L. Attention, joint attention, and social attention. Curr Dir Psychol Sci. 2007;16:269-74.

8. Posner M. Orienting of attention. Q J Exp Psychol. 1980;32:3-25.

9. Friesen $\mathrm{CK}$, Kingstone $\mathrm{A}$. The eyes have it! Reflexive orienting is triggered by nonpredictive gaze. Psychon Bull Rev. 1998;5:490-5.

10. Newport R, Howarth S. Social gaze cueing to auditory locations. Q J Exp Psychol. 2009;62:625-34.

11. Jellema T, Lorteije J, van Rijn S, van t' Wout M, de Haan E, van Engeland H, et al. Involuntary interpretation of social cues is compromised in autism spectrum disorders. Autism Res. 2009;2:192-204.

12. Senju A, Southgate $V$, White $S$, Frith U. Mindblind eyes: an absence of spontaneous theory of mind in asperger syndrome. Science. 2009:325:883-5.

13. Zhao S, Uono S, Yoshimura S, Kubota Y, Toichi M. Can gaze-cueing be helpful for detecting sound in autism spectrum disorder? Res Autism Spectr Disord. 2013;7:1250-6.

14. American Psychiatric Association [APA]. Diagnostic and statistical manual for mental disorders (DSM-IV-TR). Washington: American Psychological Association; 2000.

15. Loveland K, Landry S. Joint attention and language in autism and developmental language delay. J Autism Dev Disord. 1986;16:335-49.

16. Mundy P, Sigman M, Ungerer J, Sherman T. Defining the social deficits of autism: the contribution of nonverbal communication measures. J Child Psychol Psychiatry. 1986;27:657-69.

17. Sigman M, Ruskin E. Continuity and change in the social competence of children with autism, down dyndrome, and developmental delays. Monogr Soc Res Child Dev. 1999;64:1-114.

18. Charman T. Why is joint attention a pivotal skill in autism? Philos Trans $R$ Soc Lond B Biol Sci. 2004;358:315-24.

19. Dawson G, Toth K, Abbott R, Osterling J, Munson J, Estes A, et al. Early social attention impairments in autism: social orienting, joint attention, and attention in autism. Dev Psychol. 2004;40:271-83.

20. Barbaro J, Dissanayake C. Autism spectrum disorders in infancy and toddlerhood: a review of the evidence on early signs, early identification tools, and early diagnosis. J Dev Behav Pediatr. 2009;30:447-59.

21. Chen F, Yoon JD. Brief report: broader autism phenotype predicts spontaneous reciprocity of direct gaze. J Autism Dev Disord. 2011:41:1131-4.

22. Nummenmaa L, Engell AD, von dem Hagen E, Henson RNA, Calder AJ. Autism spectrum traits predict the neural response to eye gaze in typical individuals. Neurolmage. 2012;59:3356-63.

23. Baron-Cohen S, Wheelwright S, Skinner R, Martin J, Clubley E. The autismspectrum quotient $(\mathrm{AQ})$ : evidence from asperger syndrome/highfunctioning autism, males and females, scientists and mathematicians. J Autism Dev Disord. 2001;31:5-17.

24. Constantino JN, Todd RD. Autistic traits in the general population: a twin study. Arch Gen Psychiatry. 2003;60:524-30.

25. Bayliss AP, Pellegrino GD, Tipper SP. Sex differences in eye gaze and symbolic cueing of attention. Q J Exp Psychol. 2005;58:631-50.

26. Bayliss AP, Tipper SP. Gaze and arrow cueing of attention reveals individual differences along the autism spectrum as a function of target context. Br J Psychol. 2005:96:95-114.

27. Hudson M, Nijboer TW, Jellema T. Implicit social learning in relation to autistic traits. J Autism Dev Disord. 2012:42:2534-45.

28. Chawarska K, Klin A, Volkmar F. Automatic attention cueing through eye movement in 2-year-old children with autism. Child Dev. 2003;74:1108-22.

29. Kylliäinen $\mathrm{A}$, Hietanen JK. Attention orienting by another's gaze direction in children with autism. J Child Psychol Psychiatry. 2004;45:435-44.

30. Uono S, Sato W, Toichi M. Dynamic fearful gaze does not enhance attention orienting in individuals with Asperger's disorder. Brain Cogn. 2009;71:229-33.

31. Nation $\mathrm{K}$, Penny $\mathrm{S}$. Sensitivity to eye gaze in autism: is it normal? Is it automatic? Is it social? Dev Psychopathol. 2008;20:79-97.

32. Oldfield RC. The assessment and analysis of handedness: the Edinburgh inventory. Neuropsychologia. 1971;9:97-113.

33. Ekman P, Friesen WV. Pictures of facial affect. Palo Alto: Consulting Psychologists Press; 1976.

34. Kurita H, Osada H, Koyama T, Miyamoto Y, Kanai C, Shimizu K. Reliability and validity of the Autism Spectrum Quotient Japanese version (AQ-J). Jpn J Clin Psychiatry. 2003;32:1235-40 (in Japanese). 
35. Bayliss AP, Frischen A, Fenske MJ, Tipper SP. Affective evaluations of objects are influenced by observed gaze direction and emotional expression. Cognition. 2007:104:644-53.

36. Bayliss AP, Schuch S, Tipper SP. Gaze cueing elicited by emotional faces is influenced by affective context. Vis Cogn. 2010;18:1214-32.

37. Bonifacci P, Ricciardelli P, Lugli L, Pellicano A. Emotional attention: effects of emotion and gaze direction on overt orienting of visual attention. Cogn Process. 2007;9:127-35.

38. Fichtenholtz HM, Hopfinger JB, Graham R, Detwiler JM, LaBar KS. Happy and fearful emotion in cues and targets modulate event-related potential indices of gaze-directed attentional orienting. Soc Cogn Affect Neurosci. 2007;2:323-33

39. Friesen CK, Halvorson KM, Graham R. Emotionally meaningful targets enhance orienting triggered by a fearful gazing face. Cogn Emot. 2011;25:73-88.

40. Kuhn G, Tipples J. Increased gaze following for fearful faces. It depends on what you're looking for! Psychon Bull Rev. 2011;18:89-95.

41. Taylor T, Therrien M. Inhibition of return for the discrimination of faces. Percept Psychophys. 2008;70:279-90.

42. Pieron M, Seassau M, Leboyer M, Zalla T. Accelerated time course of saccadic inhibition of return in individuals with autism spectrum disorders. Exp Brain Res. 2015;233:767-75.

43. Mundy P, Sullivan L, Mastergeorge A. A parallel and distributed processing model of joint attention and autism. Autism Res. 2009;2:2-21.

44. Kim K, Mundy P. Joint attention, social-cognition, and recognition memory in adults. Front Hum Neurosci. 2015:6:1-11.

45. Mundy P, Jarrold W. Infant joint attention, neural networks and social cognition. Neural Netw. 2010;23:985-97.

46. Frischen A, Tipper SP. Orienting attention via observed gaze shift evokes longer term inhibitory effects: implications for social interactions, attention, and memory. J Exp Psychol Gen. 2004;133:516-33.

47. Lindemann O, Nuku P, Rueschemeyer SA, Bekkering H. Grasping the other's attention: the role of animacy in action cueing of joint attention. Vision Res. 2011;51:940-4

48. Swanson MR, Serlin GC, Sille M. Broad autism phenotype in typically developing children predicts performance on an eye-tracking measure of joint attention. J Autism Dev Disord. 2013:43:707-18.

49. Charman T, Baron-Cohen S, Swettenham J, Baird G, Cox A, Drew A. Testing joint attention, imitation, and play as infancy precursors to language and theory of mind. Cogn Dev. 2000;15:481-98.

50. Schilbach L, Wilms M, Eickhoff SB, Romanzetti S, Tepest R, Bente G, et al. Minds made for sharing: initiating joint attention recruits reward-related neurocircuitry. J Cogn Neurosci. 2010;22:2702-15.

51. Schilbach L, Eickhoff SB, Cieslik E, Shah NJ, Fink GR, Vogeley K. Eyes on me: an fMRI study of the effects of social gaze on action control. Scan. 2011;6:393-403.

52. Pfeiffer UJ, Vogeley K, Schilbach L. From gaze cueing to dual eye-tracking: novel approaches to investigate the neural correlates of gaze in social interaction. Neurosci Biobehav Rev. 2013;37:2516-28.

53. Bayliss AP, Murphy E, Naughtin CK, Kritikos A, Schilbach L, Becker SI. "Gaze leading": initiating simulated joint attention influences eye movements and choice behavior. J Exp Psychol Gen. 2013;142:76-92.

\section{Submit your next manuscript to BioMed Central and we will help you at every step:}

- We accept pre-submission inquiries

- Our selector tool helps you to find the most relevant journal

- We provide round the clock customer support

- Convenient online submission

- Thorough peer review

- Inclusion in PubMed and all major indexing services

- Maximum visibility for your research

Submit your manuscript at www.biomedcentral.com/submit

) Biomed Central 